\title{
Sparse Signal Separation in Redundant Dictionaries
}

\author{
Céline Aubel*, Christoph Studer ${ }^{\dagger}$, Graeme Pope*, and Helmut Bölcskei* \\ ${ }^{*}$ Dept. of IT \& EE, ETH Zurich, 8092 Zurich, Switzerland \\ ${ }^{\dagger}$ Dept. of ECE, Rice University, Houston, TX, USA \\ Email: \{aubelc, gpope, boelcskei\}@ nari.ee.ethz.ch, studer@ rice.edu
}

\begin{abstract}
We formulate a unified framework for the separation of signals that are sparse in "morphologically" different redundant dictionaries. This formulation incorporates the socalled "analysis" and "synthesis" approaches as special cases and contains novel hybrid setups. We find corresponding coherencebased recovery guarantees for an $\ell_{1}$-norm based separation algorithm. Our results recover those reported in Studer and Baraniuk, ACHA, submitted, for the synthesis setting, provide new recovery guarantees for the analysis setting, and form a basis for comparing performance in the analysis and synthesis settings. As an aside our findings complement the D-RIP recovery results reported in Candès et al., ACHA, 2011, for the "analysis" signal recovery problem
\end{abstract}

$$
\underset{\widetilde{\mathbf{x}}}{\operatorname{minimize}}\|\mathbf{\Psi} \widetilde{\mathbf{x}}\|_{1} \quad \text { subject to }\|\mathbf{y}-\mathbf{A} \widetilde{\mathbf{x}}\|_{2} \leq \varepsilon
$$

by delivering corresponding coherence-based recovery results.

\section{INTRODUCTION}

We consider the problem of splitting the signal $\mathbf{x}=\mathbf{x}_{1}+\mathbf{x}_{2}$ into its constituents $\mathbf{x}_{1} \in \mathbb{C}^{d}$ and $\mathbf{x}_{2} \in \mathbb{C}^{d}$-assumed to be sparse in "morphologically" different (redundant) dictionaries [1] - based on $m$ linear, nonadaptive, and noisy measurements $\mathbf{y}=\mathbf{A} \mathbf{x}+\mathbf{e}$. Here, $\mathbf{A} \in \mathbb{C}^{m \times d}, m \leq d$, is the measurement matrix, assumed to be known, and $\mathbf{e} \in \mathbb{C}^{m}$ is a noise vector, assumed to be unknown and to satisfy $\|\mathbf{e}\|_{2} \leq \varepsilon$, with $\varepsilon$ known.

Redundant dictionaries [2], [3] often lead to sparser representations than nonredundant ones, such as, e.g., orthonormal bases, and have therefore become pervasive in the sparse signal recovery literature [3]. In the context of signal separation, redundant dictionaries lead to an interesting dichotomy [1], [4], [5]:

- In the so-called "synthesis" setting, it is assumed that, for $\ell=1,2, \mathbf{x}_{\ell}=\mathbf{D}_{\ell} \mathbf{s}_{\ell}$, where $\mathbf{D}_{\ell} \in \mathbb{C}^{d \times n}(d<n)$ is a redundant dictionary (of full rank) and the coefficient vector $\mathbf{s}_{\ell} \in \mathbb{C}^{n}$ is sparse (or approximately sparse in the sense of [6]). Given the vector $y \in \mathbb{C}^{m}$, the problem of finding the constituents $\mathbf{x}_{1}$ and $\mathbf{x}_{2}$ is formalized as [7]:

$$
(\mathrm{PS}) \begin{cases}\underset{\tilde{\mathbf{s}}_{1}, \tilde{\mathbf{s}}_{2}}{\operatorname{minimize}} & \left\|\tilde{\mathbf{s}}_{1}\right\|_{1}+\left\|\tilde{\mathbf{s}}_{2}\right\|_{1} \\ \text { subject to } & \left\|\mathbf{y}-\mathbf{A}\left(\mathbf{D}_{1} \tilde{\mathbf{s}}_{1}+\mathbf{D}_{2} \tilde{\mathbf{s}}_{2}\right)\right\|_{2} \leq \varepsilon .\end{cases}
$$

- In the so-called "analysis" setting, it is assumed that, for $\ell=1,2$, there exists a matrix $\Psi_{\ell} \in \mathbb{C}^{n \times d}$ such that

The work of C. Studer was supported by the Swiss National Science Foundation (SNSF) under Grant PA00P2-134155.
$\boldsymbol{\Psi}_{\ell} \mathbf{x}_{\ell}$ is sparse (or approximately sparse). The problem of recovering $\mathbf{x}_{1}$ and $\mathbf{x}_{2}$ from $\mathbf{y}$ is formalized as [5]:

$$
(\mathrm{PA}) \begin{cases}\underset{\widetilde{\mathbf{x}}_{1}, \widetilde{\mathbf{x}}_{2}}{\operatorname{minimize}} & \left\|\boldsymbol{\Psi}_{1} \widetilde{\mathbf{x}}_{1}\right\|_{1}+\left\|\mathbf{\Psi}_{2} \widetilde{\mathbf{x}}_{2}\right\|_{1} \\ \text { subject to } & \left\|\mathbf{y}-\mathbf{A}\left(\widetilde{\mathbf{x}}_{1}+\widetilde{\mathbf{x}}_{2}\right)\right\|_{2} \leq \varepsilon .\end{cases}
$$

Throughout the paper, we exclusively consider redundant dictionaries as for $\mathbf{D}_{\ell}, \ell=1,2$, square, the synthesis setting can be recovered from the analysis setting by taking $\boldsymbol{\Psi}_{\ell}=\mathbf{D}_{\ell}^{-1}$.

The problems (PS) and (PA) arise in numerous applications including denoising [8], super-resolution [8], inpainting [9][11], deblurring [11], and recovery of sparsely corrupted signals [12]. Coherence-based recovery guarantees for (PS) were reported in [7]. The problem (PA) was mentioned in [5]. In the noiseless case, recovery guarantees for (PA), expressed in terms of a concentration inequality, are given in [13] for $\mathbf{A}=\mathbf{I}_{d}$ and $\boldsymbol{\Psi}_{1}$ and $\boldsymbol{\Psi}_{2}$ both Parseval frames [2].

Contributions: We consider the general problem

$$
(\mathrm{P}) \begin{cases}\underset{\widetilde{\mathbf{x}}_{1} \widetilde{\mathbf{x}}_{2}}{\operatorname{minimize}} & \left\|\boldsymbol{\Psi}_{1} \widetilde{\mathbf{x}}_{1}\right\|_{1}+\left\|\mathbf{\Psi}_{2} \widetilde{\mathbf{x}}_{2}\right\|_{1} \\ \text { subject to } & \left\|\mathbf{y}-\mathbf{A}_{1} \widetilde{\mathbf{x}}_{1}-\mathbf{A}_{2} \widetilde{\mathbf{x}}_{2}\right\|_{2} \leq \varepsilon,\end{cases}
$$

which encompasses (PS) and (PA). To recover (PS) from (P), one sets $\mathbf{A}_{\ell}=\mathbf{A} \mathbf{D}_{\ell}$ and $\boldsymbol{\Psi}_{\ell}=\left[\begin{array}{ll}\mathbf{I}_{d} & \mathbf{0}_{d, n-d}\end{array}\right]^{T}$, for $\ell=1,2$. (PA) is obtained by choosing $\mathbf{A}_{\ell}=\mathbf{A}$, for $\ell=1,2$. Our main contribution is a coherence-based recovery guarantee for the general problem $(\mathrm{P})$. This result recovers [7, Th. 4], which deals with (PS), provides new recovery guarantees for (PA), and constitutes a basis for comparing performance in the analysis and synthesis settings. As an aside, it also complements the D-RIP recovery guarantee in [5, Th. 1.2] for the problem

$$
\left(\mathrm{P}^{*}\right) \underset{\widetilde{\mathbf{x}}}{\operatorname{minimize}}\|\mathbf{\Psi} \widetilde{\mathbf{x}}\|_{1} \quad \text { subject to }\|\mathbf{y}-\mathbf{A} \widetilde{\mathbf{x}}\|_{2} \leq \varepsilon
$$

by delivering a corresponding coherence-based recovery guarantee. Moreover, the general formulation (P) encompasses novel hybrid problems of the form

$$
\begin{array}{ll}
\underset{\tilde{\mathbf{s}}_{1} \widetilde{\mathbf{x}}_{2}}{\operatorname{minimize}} & \left\|\tilde{\mathbf{s}}_{1}\right\|_{1}+\left\|\boldsymbol{\Psi}_{2} \widetilde{\mathbf{x}}_{2}\right\|_{1} \\
\text { subject to } & \left\|\mathbf{y}-\mathbf{A}\left(\mathbf{D}_{1} \tilde{\mathbf{s}}_{1}-\widetilde{\mathbf{x}}_{2}\right)\right\|_{2} \leq \varepsilon .
\end{array}
$$

Notation: Lowercase boldface letters stand for column vectors and uppercase boldface letters denote matrices. The transpose, conjugate transpose, and Moore-Penrose inverse of the matrix $\mathbf{M}$ are designated as $\mathbf{M}^{T}, \mathbf{M}^{H}$, and $\mathbf{M}^{\dagger}$, respectively. The $j$ th column of $\mathbf{M}$ is written $[\mathbf{M}]_{j}$, and 


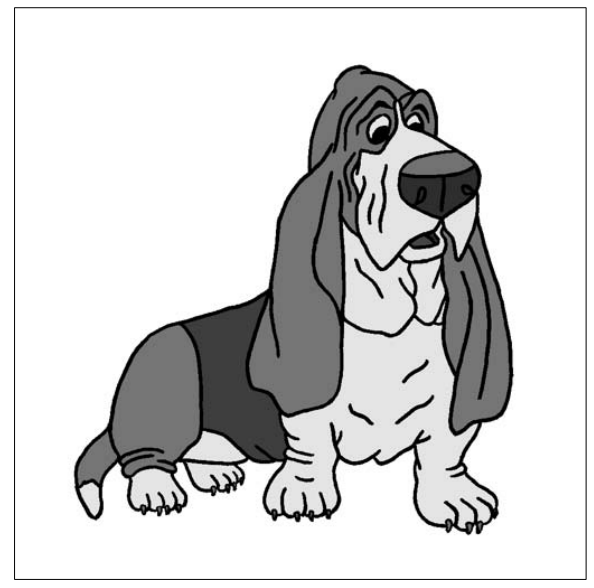

(a) Original cartoon image

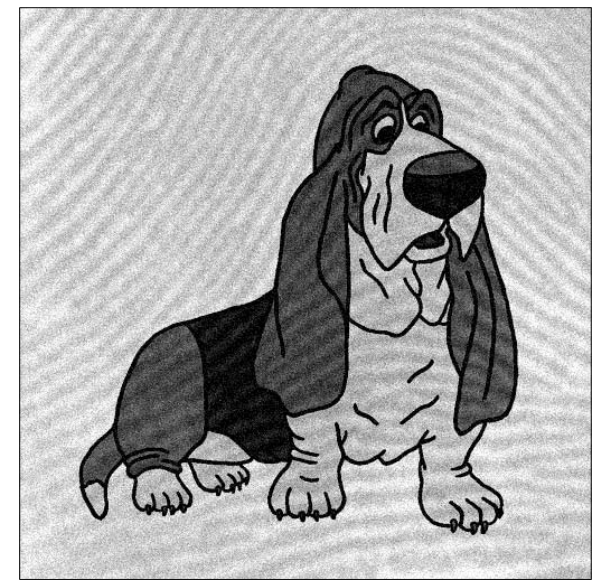

(b) Corrupted image

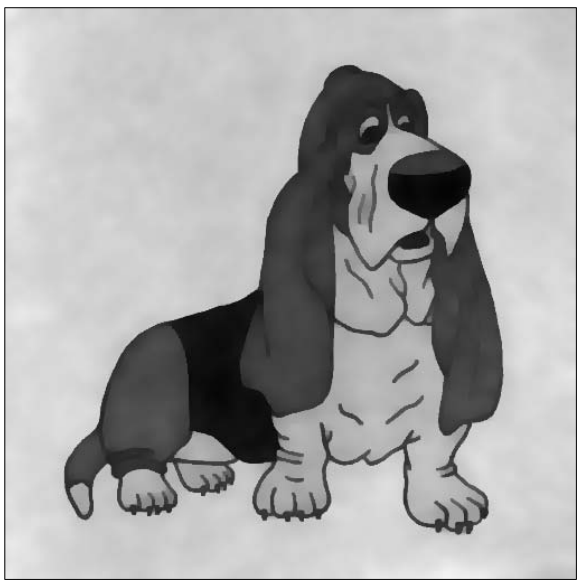

(c) Restored cartoon image

Fig. 1: Image separation in the presence of Gaussian noise ( $\mathrm{SNR}=20 \mathrm{~dB})$.

the entry in the $i$ th row and $j$ th column of $\mathbf{M}$ is $[\mathbf{M}]_{i, j}$. We let $\sigma_{\min }(\mathbf{M})$ denote the smallest singular value of $\mathbf{M}$, use $\mathbf{I}_{n}$ for the $n \times n$ identity matrix, and let $\mathbf{0}_{k \times m}$ be the $k \times m$ all zeros matrix. For matrices $\mathbf{M}$ and $\mathbf{N}$, we let $\omega_{\min }(\mathbf{M}) \triangleq \min _{j}\left\|[\mathbf{M}]_{j}\right\|_{2}, \omega_{\max }(\mathbf{M}) \triangleq \max _{j}\left\|[\mathbf{M}]_{j}\right\|_{2}$, $\omega_{\min }(\mathbf{M}, \mathbf{N}) \triangleq \min \left\{\omega_{\min }(\mathbf{M}), \omega_{\min }(\mathbf{N})\right\}$, and $\omega_{\max }(\mathbf{M}, \mathbf{N}) \triangleq$ $\max \left\{\omega_{\max }(\mathbf{M}), \omega_{\max }(\mathbf{N})\right\}$. The $k$ th entry of the vector $\mathbf{x}$ is written $[\mathbf{x}]_{k}$, and $\|\mathbf{x}\|_{1} \triangleq \sum_{k}\left|[\mathbf{x}]_{k}\right|$ stands for its $\ell_{1}$-norm. We take $\operatorname{supp}_{k}(\mathbf{x})$ to be the set of indices corresponding to the $k$ largest (in magnitude) coefficients of $\mathbf{x}$. Sets are designated by uppercase calligraphic letters; the cardinality of the set $\mathcal{S}$ is $|\mathcal{S}|$ and the complement of $\mathcal{S}$ (in some given set) is denoted by $\mathcal{S}^{c}$. For a set $\mathcal{S}$ of integers and $n \in \mathbb{Z}$, we let $n+\mathcal{S} \triangleq\{n+p: p \in \mathcal{S}\}$. The $n \times n$ diagonal projection matrix $\mathbf{P}_{\mathcal{S}}$ for the set $\mathcal{S} \subset\{1, \ldots, n\}$ is defined as follows:

$$
\left[\mathbf{P}_{\mathcal{S}}\right]_{i, j}= \begin{cases}1, & i=j \text { and } i \in \mathcal{S} \\ 0, & \text { otherwise, }\end{cases}
$$

and we set $\mathbf{M}_{\mathcal{S}} \triangleq \mathbf{P}_{\mathcal{S}} \mathbf{M}$. We define $\sigma_{k}(\mathbf{x})$ to be the $\ell_{1}$-norm approximation error of the best $k$-sparse approximation of $\mathbf{x}$, i.e., $\sigma_{k}(\mathbf{x}) \triangleq\left\|\mathbf{x}-\mathbf{x}_{\mathcal{S}}\right\|_{1}$ where $\mathcal{S}=\operatorname{supp}_{k}(\mathbf{x})$ and $\mathbf{x}_{\mathcal{S}} \triangleq \mathbf{P}_{\mathcal{S}} \mathbf{x}$.

\section{RECOVERY GuARANTEES}

Coherence definitions in the sparse signal recovery literature [3] usually apply to dictionaries with normalized columns. Here, we will need coherence notions valid for general (unnormalized) dictionaries $\mathbf{M}$ and $\mathbf{N}$, assumed, for simplicity of exposition, to consist of nonzero columns only.

Definition 1 (Coherence): The coherence of the dictionary $\mathbf{M}$ is defined as

$$
\hat{\mu}(\mathbf{M})=\max _{i, j, i \neq j} \frac{\left|\left[\mathbf{M}^{H} \mathbf{M}\right]_{i, j}\right|}{\omega_{\min }^{2}(\mathbf{M})} .
$$

Definition 2 (Mutual coherence): The mutual coherence of the dictionaries $\mathbf{M}$ and $\mathbf{N}$ is defined as

$$
\hat{\mu}_{m}(\mathbf{M}, \mathbf{N})=\max _{i, j} \frac{\left|\left[\mathbf{M}^{H} \mathbf{N}\right]_{i, j}\right|}{\omega_{\min }^{2}(\mathbf{M}, \mathbf{N})}
$$

The main contribution of this paper is the following recovery guarantee for $(\mathrm{P})$.

Theorem 1: Let $\mathbf{y}=\mathbf{A}_{1} \mathbf{x}_{1}+\mathbf{A}_{2} \mathbf{x}_{2}+\mathbf{e}$ with $\|\mathbf{e}\|_{2} \leq \varepsilon$ and let $\boldsymbol{\Psi}_{1} \in \mathbb{C}^{n_{1} \times p_{1}}$ and $\boldsymbol{\Psi}_{2} \in \mathbb{C}^{n_{2} \times p_{2}}$ be full-rank matrices. Let $\mathbf{x}=\left[\mathbf{x}_{1}^{T} \mathbf{x}_{2}^{T}\right]^{T}, \hat{\mu}_{1}=\hat{\mu}\left(\mathbf{A}_{1} \Psi_{1}^{\dagger}\right), \hat{\mu}_{2}=\hat{\mu}\left(\mathbf{A}_{2} \mathbf{\Psi}_{2}^{\dagger}\right), \hat{\mu}_{m}=$ $\hat{\mu}_{m}\left(\mathbf{A}_{1} \mathbf{\Psi}_{1}^{\dagger}, \mathbf{A}_{2} \mathbf{\Psi}_{2}^{\dagger}\right)$, and $\hat{\mu}_{\max }=\max \left\{\hat{\mu}_{1}, \hat{\mu}_{2}, \hat{\mu}_{m}\right\}$. Without loss of generality, we assume that $\hat{\mu}_{1} \leq \hat{\mu}_{2}$. Let $k_{1}$ and $k_{2}$ be nonnegative integers such that

$$
k_{1}+k_{2}<\max \left\{\frac{2\left(1+\hat{\mu}_{2}\right)}{\hat{\mu}_{2}+2 \hat{\mu}_{\max }+\sqrt{\hat{\mu}_{2}^{2}+\hat{\mu}_{m}^{2}}}, \frac{1+\hat{\mu}_{\max }}{2 \hat{\mu}_{\max }}\right\} \text {. }
$$

Then, the solution $\left(\mathrm{x}_{1}^{*}, \mathrm{x}_{2}^{*}\right)$ to the convex program $(\mathrm{P})$ satisfies

$$
\left\|\mathbf{x}^{*}-\mathbf{x}\right\|_{2} \leq C_{0} \varepsilon+C_{1}\left(\sigma_{k_{1}}\left(\mathbf{\Psi}_{1} \mathbf{x}_{1}\right)+\sigma_{k_{2}}\left(\mathbf{\Psi}_{2} \mathbf{x}_{2}\right)\right),
$$

where $C_{0}, C_{1} \geq 0$ are constants that do not depend on $\mathbf{x}_{1}$ and $\mathbf{x}_{2}$ and where $\mathbf{x}^{*}=\left[\begin{array}{ll}\mathbf{x}_{1}^{* T} & \mathbf{x}_{2}^{* T}\end{array}\right]^{T}$.

Note that the quantities $\hat{\mu}_{1}, \hat{\mu}_{2}$, and $\hat{\mu}_{m}$ characterize the interplay between the measurement matrix $\mathbf{A}$ and the sparsifying transforms $\boldsymbol{\Psi}_{1}$ and $\boldsymbol{\Psi}_{2}$.

As a corollary to our main result, we get the following statement for the problem $\left(\mathrm{P}^{*}\right)$ considered in [5].

Corollary 2: Let $\mathbf{y}=\mathbf{A x}+\mathbf{e}$ with $\|\mathbf{e}\|_{2} \leq \varepsilon$ and let $\mathbf{\Psi} \in$ $\mathbb{C}^{n \times p}$ be a full-rank matrix. Let $k$ be a nonnegative integer such that

$$
k<\frac{1}{2}\left(1+\frac{1}{\hat{\mu}\left(\mathbf{A} \Psi^{\dagger}\right)}\right) .
$$

Then, the solution $\mathrm{x}^{*}$ to the convex program $\left(\mathrm{P}^{*}\right)$ satisfies

$$
\left\|\mathbf{x}^{*}-\mathbf{x}\right\|_{2} \leq C_{0} \varepsilon+C_{1} \sigma_{k}(\mathbf{\Psi} \mathbf{x})
$$

where $C_{0}, C_{1} \geq 0$ are constants ${ }^{1}$ that do not depend on $\mathbf{x}$.

The proofs of Theorem 1 and Corollary 2 can be found in the Appendix.

We conclude by noting that D-RIP recovery guarantees for $\left(\mathrm{P}^{*}\right)$ were provided in [5]. As is common in RIP-based

${ }^{1}$ Note that the constants $C_{0}$ and $C_{1}$ may take on different values at each occurrence. 
recovery guarantees the restricted isometry constants are, in general, hard to compute. Moreover, the results in [5] hinge on the assumption that $\boldsymbol{\Psi}$ forms a Parseval frame, i.e., $\Psi^{H} \boldsymbol{\Psi}=\mathbf{I}_{d}$; a corresponding extension to general $\boldsymbol{\Psi}$ was provided in [14]. We finally note that it does not seem possible to infer the coherence-based threshold (5) from the D-RIP recovery guarantees in [5], [14].

\section{NUMERICAL RESULTS}

We analyze an image-separation problem where we remove a fingerprint from a cartoon image. We corrupt the $512 \times 512$ greyscale cartoon image depicted in Fig. 1(a) by adding a fingerprint $^{2}$ and i.i.d. zero-mean Gaussian noise.

Cartoon images are constant apart from (a small number of) discontinuities and are thus sparse under the finite difference operator $\boldsymbol{\Delta}$ defined in [15]. Fingerprints are sparse under the application of a wave atom transform, $\mathbf{W}$, such as the redundancy 2 transform available in the WaveAtom toolbox ${ }^{3}$ [16]. It is therefore sensible to perform separation by solving the problem (PA) with $\boldsymbol{\Psi}_{1}=\boldsymbol{\Delta}, \boldsymbol{\Psi}_{2}=\mathbf{W}$, and $\mathbf{A}=\mathbf{I}_{d}$. For our simulation, we use a regularized version of $\Delta$ and we employ the TFOCS solver ${ }^{4}$ from [17].

Fig. 1(c) shows the corresponding recovered image. We can see that the restoration procedure gives visually satisfactory results.

\section{APPENDiX A \\ PROOFS}

For simplicity of exposition, we first present the proof of Corollary 2 and then describe the proof of Theorem 1 .

\section{A. Proof of Corollary 2}

We define the vector $\mathbf{h}=\mathbf{x}^{*}-\mathbf{x}$, where $\mathrm{x}^{*}$ is the solution to $\left(\mathrm{P}^{*}\right)$ and $\mathrm{x}$ is the vector to be recovered. We furthermore set $\mathcal{S}=\operatorname{supp}_{k}\left(\Psi_{\mathbf{X}}\right)$.

1) Prerequisites: Our proof relies partly on two important results developed earlier in [5], [6] and summarized, for completeness, next.

Lemma 3 (Cone constraint [5], [6]): The vector $\boldsymbol{\Psi h}$ obeys

$$
\left\|\boldsymbol{\Psi}_{\mathcal{S}^{c}} \mathbf{h}\right\|_{1} \leq\left\|\boldsymbol{\Psi}_{\mathcal{S}} \mathbf{h}\right\|_{1}+2\left\|\boldsymbol{\Psi}_{\mathcal{S}^{c}} \mathbf{X}\right\|_{1}
$$

where $\mathcal{S}=\operatorname{supp}_{k}\left(\boldsymbol{\Psi}_{\mathbf{x}}\right)$.

Proof: Since $\mathrm{x}^{*}$ is the minimizer of $\left(\mathrm{P}^{*}\right)$, the inequality $\left\|\boldsymbol{\Psi}_{\mathbf{X}}\right\|_{1} \geq\left\|\boldsymbol{\Psi}_{\mathbf{X}^{*}}\right\|_{1}$ holds. Using $\boldsymbol{\Psi}=\boldsymbol{\Psi}_{\mathcal{S}}+\boldsymbol{\Psi}_{\mathcal{S}^{c}}$ and $\mathbf{x}^{*}=$ $\mathbf{x}+\mathbf{h}$, we obtain

$$
\begin{aligned}
& \left\|\boldsymbol{\Psi}_{\mathcal{S}} \mathbf{x}\right\|_{1}+\left\|\boldsymbol{\Psi}_{\mathcal{S}^{c}} \mathbf{x}\right\|_{1}=\left\|\Psi_{\mathbf{x}}\right\|_{1} \\
& \geq\left\|\boldsymbol{\Psi}_{\mathbf{x}^{*}}\right\|_{1}=\left\|\boldsymbol{\Psi}_{\mathcal{S}} \mathbf{x}+\boldsymbol{\Psi}_{\mathcal{S}} \mathbf{h}\right\|_{1}+\left\|\boldsymbol{\Psi}_{\mathcal{S}^{c} \mathbf{x}}+\boldsymbol{\Psi}_{\mathcal{S}^{c}} \mathbf{h}\right\|_{1} \\
& \geq\left\|\boldsymbol{\Psi}_{\mathcal{S}} \mathbf{x}\right\|_{1}-\left\|\boldsymbol{\Psi}_{\mathcal{S}} \mathbf{h}\right\|_{1}+\left\|\boldsymbol{\Psi}_{\mathcal{S}^{c}} \mathbf{h}\right\|_{1}-\left\|\boldsymbol{\Psi}_{\mathcal{S}^{c} \mathbf{X}}\right\|_{1} \text {. }
\end{aligned}
$$

We retrieve (7) by simple rearrangement of terms.

Lemma 4 (Tube constraint [5], [6]): The vector Ah satisfies $\|\mathbf{A h}\|_{2} \leq 2 \varepsilon$.

\footnotetext{
${ }^{2}$ The fingerprint image is taken from http://commons.wikimedia.org/

${ }^{3}$ We used the WaveAtom toolbox from http://www. waveatom.org/

${ }^{4}$ We used TFOCS from http://tfocs.stanford.edu/
}

Proof: Since both $\mathrm{x}^{*}$ and $\mathrm{x}$ are feasible (we recall that $\mathbf{y}=\mathbf{A} \mathbf{x}+\mathbf{e}$ with $\left.\|\mathbf{e}\|_{2} \leq \varepsilon\right)$, we have the following

$$
\begin{aligned}
\|\mathbf{A h}\|_{2} & =\left\|\mathbf{A}\left(\mathbf{x}^{*}-\mathbf{x}\right)\right\|_{2} \\
& \leq\left\|\mathbf{A} \mathbf{x}^{*}-\mathbf{y}\right\|_{2}+\|\mathbf{y}-\mathbf{A} \mathbf{x}\|_{2} \leq 2 \varepsilon,
\end{aligned}
$$

thus establishing the lemma.

2) Bounding the recovery error: We want to bound $\|\mathbf{h}\|_{2}$ from above. Since $\sigma_{\min }(\boldsymbol{\Psi})>0$ by assumption ( $\boldsymbol{\Psi}$ is assumed to be full-rank), it follows from the Rayleigh-Ritz theorem [18, Th. 4.2.2] that

$$
\|\mathbf{h}\|_{2} \leq \frac{1}{\sigma_{\min }(\boldsymbol{\Psi})}\|\boldsymbol{\Psi} \mathbf{h}\|_{2} .
$$

We now set $\mathcal{Q}=\operatorname{supp}_{k}(\boldsymbol{\Psi h})$. Clearly, we have for $i \in \mathcal{Q}^{c}$,

$$
\left|[\boldsymbol{\Psi} \mathbf{h}]_{i}\right| \leq \frac{\left\|\boldsymbol{\Psi}_{\mathcal{Q}} \mathbf{h}\right\|_{1}}{k} .
$$

Using the same argument as in [19, Th. 3.1], we obtain

$$
\begin{aligned}
\left\|\boldsymbol{\Psi}_{\mathcal{Q}^{c}} \mathbf{h}\right\|_{2}^{2}=\sum_{i \in \mathcal{Q}^{c}}\left|[\boldsymbol{\Psi} \mathbf{h}]_{i}\right|^{2} & \leq \sum_{i \in \mathcal{Q}^{c}}\left|[\boldsymbol{\Psi} \mathbf{h}]_{i}\right| \frac{\left\|\boldsymbol{\Psi}_{\mathcal{Q}} \mathbf{h}\right\|_{1}}{k} \\
& =\left\|\boldsymbol{\Psi}_{\mathcal{Q}^{c}} \mathbf{h}\right\|_{1} \frac{\left\|\boldsymbol{\Psi}_{\mathcal{Q}} \mathbf{h}\right\|_{1}}{k}
\end{aligned}
$$

Since $\mathcal{Q}$ is the set of indices of the $k$ largest (in magnitude) coefficients of $\boldsymbol{\Psi h}$ and since $\mathcal{Q}$ and $\mathcal{S}$ both contain $k$ elements, we have $\left\|\boldsymbol{\Psi}_{\mathcal{S}} \mathbf{h}\right\|_{1} \leq\left\|\boldsymbol{\Psi}_{\mathcal{Q}} \mathbf{h}\right\|_{1}$ and $\left\|\boldsymbol{\Psi}_{\mathcal{Q}^{c}} \mathbf{h}\right\|_{1} \leq\left\|\boldsymbol{\Psi}_{\mathcal{S}^{c}} \mathbf{h}\right\|_{1}$, which, combined with the cone constraint in Lemma 3, yields

$$
\left\|\boldsymbol{\Psi}_{\mathcal{Q}^{c}} \mathbf{h}\right\|_{1} \leq\left\|\boldsymbol{\Psi}_{\mathcal{Q}} \mathbf{h}\right\|_{1}+2\left\|\boldsymbol{\Psi}_{\mathcal{S}^{c}} \mathbf{X}\right\|_{1}
$$

The inequality in (10) then becomes

$$
\begin{aligned}
\left\|\boldsymbol{\Psi}_{\mathcal{Q}^{c}} \mathbf{h}\right\|_{2}^{2} & \leq \frac{\left\|\boldsymbol{\Psi}_{\mathcal{Q}} \mathbf{h}\right\|_{1}^{2}}{k}+2\left\|\boldsymbol{\Psi}_{\mathcal{S}^{c} \mathbf{x}}\right\|_{1} \frac{\left\|\boldsymbol{\Psi}_{\mathcal{Q}} \mathbf{h}\right\|_{1}}{k} \\
& \leq\left\|\boldsymbol{\Psi}_{\mathcal{Q}} \mathbf{h}\right\|_{2}^{2}+2\left\|\boldsymbol{\Psi}_{\mathcal{S}^{c} \mathbf{X}}\right\|_{1} \frac{\left\|\boldsymbol{\Psi}_{\mathcal{Q}} \mathbf{h}\right\|_{2}}{\sqrt{k}} \\
& \leq 2\left\|\boldsymbol{\Psi}_{\mathcal{Q}} \mathbf{h}\right\|_{2}^{2}+\frac{\left\|\boldsymbol{\Psi}_{\mathcal{S}^{c} \mathbf{X}}\right\|_{1}^{2}}{k}
\end{aligned}
$$

where (12a) follows from $\|\mathbf{u}\|_{1} \leq \sqrt{k}\|\mathbf{u}\|_{2}$ for $k$-sparse ${ }^{5} \mathbf{u}$ and $(12 \mathrm{~b})$ is a consequence of $2 x y \leq x^{2}+y^{2}$, for $x, y \in \mathbb{R}$.

It now follows that

$$
\begin{aligned}
\|\boldsymbol{\Psi} \mathbf{h}\|_{2} & =\sqrt{\left\|\boldsymbol{\Psi}_{\mathcal{Q}} \mathbf{h}\right\|_{2}^{2}+\left\|\boldsymbol{\Psi}_{\mathcal{Q}^{c}} \mathbf{h}\right\|_{2}^{2}} \\
& \leq \sqrt{3\left\|\boldsymbol{\Psi}_{\mathcal{Q}} \mathbf{h}\right\|_{2}^{2}+\frac{\left\|\boldsymbol{\Psi}_{\mathcal{S}^{c}} \mathbf{x}\right\|_{1}^{2}}{k}} \\
& \leq \sqrt{3}\left\|\boldsymbol{\Psi}_{\mathcal{Q}} \mathbf{h}\right\|_{2}+\frac{\left\|\boldsymbol{\Psi}_{\mathcal{S}^{c} \mathbf{x}}\right\|_{1}}{\sqrt{k}}
\end{aligned}
$$

where (13a) is a consequence of (12b) and (13b) results from $\sqrt{x^{2}+y^{2}} \leq x+y$, for $x, y \geq 0$.

Combining (9) and (13b) leads to

$$
\|\mathbf{h}\|_{2} \leq \frac{1}{\sigma_{\min }(\boldsymbol{\Psi})}\left(\sqrt{3}\left\|\boldsymbol{\Psi}_{\mathcal{Q}} \mathbf{h}\right\|_{2}+\frac{\left\|\boldsymbol{\Psi}_{\mathcal{S}^{c}} \mathbf{X}\right\|_{1}}{\sqrt{k}}\right) .
$$

${ }^{5} \mathrm{~A}$ vector is $k$-sparse if it has at most $k$ nonzero entries. 
3) Bounding the term $\left\|\boldsymbol{\Psi}_{\mathcal{Q}} \mathbf{h}\right\|_{2}$ in (14): In the last step of the proof, we bound the term $\left\|\Psi_{\mathcal{Q}} \mathbf{h}\right\|_{2}$ in (14). To this end, we first bound $\left\|\mathbf{A} \boldsymbol{\Psi}^{\dagger} \boldsymbol{\Psi}_{\mathcal{Q}} \mathbf{h}\right\|_{2}^{2}$, with $\boldsymbol{\Psi}^{\dagger}=\left(\boldsymbol{\Psi}^{H} \boldsymbol{\Psi}\right)^{-1} \boldsymbol{\Psi}^{H}$, using Geršgorin's disc theorem [18, Th. 6.2.2]:

$$
\theta_{\min }\left\|\boldsymbol{\Psi}_{\mathcal{Q}} \mathbf{h}\right\|_{2}^{2} \leq\left\|\mathbf{A} \boldsymbol{\Psi}^{\dagger} \boldsymbol{\Psi}_{\mathcal{Q}} \mathbf{h}\right\|_{2}^{2} \leq \theta_{\max }\left\|\boldsymbol{\Psi}_{\mathcal{Q}} \mathbf{h}\right\|_{2}^{2}
$$

where $\theta_{\min } \triangleq \omega_{\min }^{2}-\mu(k-1)$ and $\theta_{\max } \triangleq \omega_{\max }^{2}+\mu(k-1)$ with

$$
\mu=\max _{i, j, i \neq j}\left|\left[\left(\mathbf{A} \boldsymbol{\Psi}^{\dagger}\right)^{H} \mathbf{A} \boldsymbol{\Psi}^{\dagger}\right]_{i, j}\right|
$$

and $\omega_{\min } \triangleq \omega_{\min }\left(\mathbf{A} \Psi^{\dagger}\right)$ and $\omega_{\max } \triangleq \omega_{\max }\left(\mathbf{A} \Psi^{\dagger}\right)$.

Using Lemma 4 and (15) and following the same steps as in [20, Th. 2.1] and [7, Th. 1], we arrive at the following chain of inequalities:

$$
\begin{aligned}
& \theta_{\min }\left\|\boldsymbol{\Psi}_{\mathcal{Q}} \mathbf{h}\right\|_{2}^{2} \leq\left\|\mathbf{A} \boldsymbol{\Psi}^{\dagger} \boldsymbol{\Psi}_{\mathcal{Q}} \mathbf{h}\right\|_{2}^{2}=\left(\mathbf{A} \boldsymbol{\Psi}^{\dagger} \boldsymbol{\Psi}_{\mathcal{Q}} \mathbf{h}\right)^{H} \mathbf{A} \boldsymbol{\Psi}^{\dagger} \boldsymbol{\Psi}_{\mathcal{Q}} \mathbf{h} \\
& =(\mathbf{A} \mathbf{h})^{H} \mathbf{A} \boldsymbol{\Psi}^{\dagger} \boldsymbol{\Psi}_{\mathcal{Q}} \mathbf{h}-\left(\mathbf{A} \boldsymbol{\Psi}^{\dagger} \boldsymbol{\Psi}_{\mathcal{Q}^{c}} \mathbf{h}\right)^{H} \mathbf{A} \boldsymbol{\Psi}^{\dagger} \boldsymbol{\Psi}_{\mathcal{Q}} \mathbf{h} \\
& \leq\left|(\mathbf{A} \mathbf{h})^{H} \mathbf{A} \boldsymbol{\Psi}^{\dagger} \boldsymbol{\Psi}_{\mathcal{Q}} \mathbf{h}\right|+\left|\left(\boldsymbol{\Psi}_{\mathcal{Q}^{c}} \mathbf{h}\right)^{H}\left(\mathbf{A} \boldsymbol{\Psi}^{\dagger}\right)^{H} \mathbf{A} \boldsymbol{\Psi}^{\dagger}\left(\boldsymbol{\Psi}_{\mathcal{Q}} \mathbf{h}\right)\right| \\
& \leq\|\mathbf{A h}\|_{2}\left\|\mathbf{A} \boldsymbol{\Psi}^{\dagger} \Psi_{\mathcal{Q}} \mathbf{h}\right\|_{2} \\
& +\sum_{i \in \mathcal{Q}^{c}, j \in \mathcal{Q}}\left|\left[\left(\mathbf{A} \boldsymbol{\Psi}^{\dagger}\right)^{H} \mathbf{A} \boldsymbol{\Psi}^{\dagger}\right]_{i, j}\right|\left|[\mathbf{\Psi} \mathbf{h}]_{i} \|[\mathbf{\Psi} \mathbf{h}]_{j}\right| \\
& \leq 2 \varepsilon \sqrt{\theta_{\max }}\left\|\boldsymbol{\Psi}_{\mathcal{Q}} \mathbf{h}\right\|_{2}+\mu\left\|\boldsymbol{\Psi}_{\mathcal{Q}} \mathbf{h}\right\|_{1}\left\|\boldsymbol{\Psi}_{\mathcal{Q}^{c}} \mathbf{h}\right\|_{1} \\
& \leq 2 \varepsilon \sqrt{\theta_{\max }}\left\|\boldsymbol{\Psi}_{\mathcal{Q}} \mathbf{h}\right\|_{2}+\mu\left\|\boldsymbol{\Psi}_{\mathcal{Q}} \mathbf{h}\right\|_{1}\left(\left\|\boldsymbol{\Psi}_{\mathcal{Q}} \mathbf{h}\right\|_{1}+2\left\|\boldsymbol{\Psi}_{\mathcal{S}^{c} \mathbf{x}}\right\|_{1}\right) \\
& \leq 2 \varepsilon \sqrt{\theta_{\max }}\left\|\boldsymbol{\Psi}_{\mathcal{Q}} \mathbf{h}\right\|_{2}+\mu k\left\|\boldsymbol{\Psi}_{\mathcal{Q}} \mathbf{h}\right\|_{2}^{2} \\
& +2 \mu \sqrt{k}\left\|\boldsymbol{\Psi}_{\mathcal{S}^{c}} \mathbf{x}\right\|_{1}\left\|\boldsymbol{\Psi}_{\mathcal{Q}} \mathbf{h}\right\|_{2}
\end{aligned}
$$

where (17a) follows from $\Psi_{\mathcal{Q}} \mathbf{h}=\boldsymbol{\Psi} \mathbf{h}-\boldsymbol{\Psi}_{\mathcal{Q}^{c}} \mathbf{h}$ and $\boldsymbol{\Psi}^{\dagger} \boldsymbol{\Psi}=$ $\mathbf{I}_{d},(17 \mathrm{~b})$ is a consequence of the Cauchy-Schwarz inequality, (17c) is obtained from (15), Lemma 4, and the definition of $\mu$ in (16), (17d) results from (11), and (17e) comes from $\|\mathbf{u}\|_{1} \leq$ $\sqrt{k}\|\mathbf{u}\|_{2}$, for $k$-sparse $\mathbf{u}$.

If $\mathbf{h} \neq \mathbf{0}$, then $\left\|\boldsymbol{\Psi}_{\mathcal{Q}} \mathbf{h}\right\|_{2} \neq 0$, since $\boldsymbol{\Psi}$ is assumed to be full-rank and $\mathcal{Q}$ is the set of indices of the $k$ largest (in magnitude) coefficients of $\boldsymbol{\Psi h}$, and therefore, the inequality between $\theta_{\min }\left\|\boldsymbol{\Psi}_{\mathcal{Q}} \mathbf{h}\right\|_{2}^{2}$ and (17e) simplifies to

$$
\left(\omega_{\min }^{2}-\mu(2 k-1)\right)\left\|\boldsymbol{\Psi}_{\mathcal{Q}} \mathbf{h}\right\|_{2} \leq 2 \varepsilon \sqrt{\theta_{\max }}+2 \mu \sqrt{k}\left\|\boldsymbol{\Psi}_{\mathcal{S}^{c}} \mathbf{x}\right\|_{1} .
$$

This finally yields

$$
\left\|\boldsymbol{\Psi}_{\mathcal{Q}} \mathbf{h}\right\|_{2} \leq \frac{2 \varepsilon \sqrt{\theta_{\max }}+2 \mu \sqrt{k}\left\|\boldsymbol{\Psi}_{\mathcal{S}^{c} \mathbf{x}}\right\|_{1}}{\omega_{\min }^{2}-\mu(2 k-1)}
$$

provided that

$$
\omega_{\min }^{2}-\mu(2 k-1)>0 .
$$

4) Recovery guarantee: Using Definition 1, we get $\hat{\mu}=$ $\hat{\mu}\left(\mathbf{A} \Psi^{\dagger}\right)=\mu / \omega_{\min }^{2}$. Combining (14) and (18), we therefore conclude that for

$$
k<\frac{1}{2}\left(1+\frac{1}{\hat{\mu}}\right)
$$

we have

$$
\left\|\mathbf{x}^{*}-\mathbf{x}\right\|_{2}=\|\mathbf{h}\|_{2} \leq C_{0} \varepsilon+C_{1}\left\|\Psi_{\mathcal{S}^{c}} \mathbf{x}\right\|_{1}
$$

with

$$
\begin{aligned}
C_{0} & =\frac{2 \sqrt{3}}{\sigma_{\min }(\boldsymbol{\Psi}) \omega_{\min }} \frac{\sqrt{\frac{\omega_{\max }^{2}}{\omega_{\min }^{2}}(1+\hat{\mu}(k-1))}}{1-\hat{\mu}(2 k-1)} \\
C_{1} & =\frac{1}{\sigma_{\min }(\boldsymbol{\Psi})}\left(\frac{2 \hat{\mu} \sqrt{3 k}}{1-\hat{\mu}(2 k-1)}+\frac{1}{\sqrt{k}}\right) .
\end{aligned}
$$

\section{B. Proof of Theorem 1}

We start by transforming $(\mathrm{P})$ into the equivalent problem $\left(\mathrm{P}^{*}\right) \underset{\widetilde{\mathbf{x}}}{\operatorname{minimize}}\|\boldsymbol{\Psi} \widetilde{\mathbf{x}}\|_{1} \quad$ subject to $\|\mathbf{y}-\mathbf{A} \widetilde{\mathbf{x}}\|_{2} \leq \varepsilon$ by amalgamating $\boldsymbol{\Psi}_{1}, \boldsymbol{\Psi}_{2}$ and $\mathbf{A}_{1}, \mathbf{A}_{2}$ into the matrices $\boldsymbol{\Psi}$ and A as follows:

$$
\begin{aligned}
& \mathbf{A}=\left[\begin{array}{ll}
\mathbf{A}_{1} & \mathbf{A}_{2}
\end{array}\right] \in \mathbb{C}^{m \times p} \\
& \boldsymbol{\Psi}=\left[\begin{array}{cc}
\boldsymbol{\Psi}_{1} & \mathbf{0}_{n \times d} \\
\mathbf{0}_{n \times d} & \boldsymbol{\Psi}_{2}
\end{array}\right] \in \mathbb{C}^{2 n \times 2 d},
\end{aligned}
$$

where $p=2 d$ in the analysis setting, $p=2 n$ in the synthesis setting, and $p=d+n$ in hybrid settings. The corresponding measurement vector is $\mathbf{y}=\mathbf{A x}+\mathbf{e}$, where we set $\mathbf{x}=\left[\begin{array}{ll}\mathbf{x}_{1}^{T} & \mathbf{x}_{2}^{T}\end{array}\right]^{T}$.

A recovery condition for $(\mathrm{P})$ could now be obtained by simply inserting $\mathbf{A}$ and $\boldsymbol{\Psi}$ in (20), (21) above into (5). In certain cases, we can, however, get a better (i.e., less restrictive) threshold following ideas similar to those reported in [7] and detailed next.

We define the vectors $\mathbf{h}_{1}=\mathbf{x}_{1}^{*}-\mathbf{x}_{1}, \mathbf{h}_{2}=\mathbf{x}_{2}^{*}-\mathbf{x}_{2}$, the sets $\mathcal{Q}_{1} \triangleq \operatorname{supp}_{k_{1}}\left(\boldsymbol{\Psi}_{1} \mathbf{h}_{1}\right), \mathcal{Q}_{2} \triangleq n+\operatorname{supp}_{k_{2}}\left(\boldsymbol{\Psi}_{2} \mathbf{h}_{2}\right)$, and $\mathbf{h}=\left[\mathbf{h}_{1}^{T} \mathbf{h}_{2}^{T}\right]^{T}, \mathcal{Q}=\mathcal{Q}_{1} \cup \mathcal{Q}_{2}$, and set $k=k_{1}+k_{2}$.

We furthermore let, for $\ell=1,2$,

$$
\begin{aligned}
& \mu_{\ell}=\max _{i, j, i \neq j}\left|\left[\left(\mathbf{A}_{\ell} \boldsymbol{\Psi}_{\ell}^{\dagger}\right)^{H} \mathbf{A}_{\ell} \boldsymbol{\Psi}_{\ell}^{\dagger}\right]_{i, j}\right| \\
& \mu_{m}=\max _{i, j}\left|\left[\left(\mathbf{A}_{1} \boldsymbol{\Psi}_{1}^{\dagger}\right)^{H} \mathbf{A}_{2} \boldsymbol{\Psi}_{2}^{\dagger}\right]_{i, j}\right| .
\end{aligned}
$$

With the definitions of $\mathcal{Q}_{1}$ and $\mathcal{Q}_{2}$, we have from (15)

$$
\begin{array}{r}
\left\|\mathbf{A} \boldsymbol{\Psi}^{\dagger} \boldsymbol{\Psi}_{\mathcal{Q}} \mathbf{h}\right\|_{2}^{2}=\left\|\mathbf{A} \boldsymbol{\Psi}^{\dagger} \boldsymbol{\Psi}_{\mathcal{Q}_{1}} \mathbf{h}\right\|_{2}^{2}+\left\|\mathbf{A} \boldsymbol{\Psi}^{\dagger} \boldsymbol{\Psi}_{\mathcal{Q}_{2}} \mathbf{h}\right\|_{2}^{2} \\
+2\left(\mathbf{A} \boldsymbol{\Psi}^{\dagger} \boldsymbol{\Psi}_{\mathcal{Q}_{1}} \mathbf{h}\right)^{H} \mathbf{A} \boldsymbol{\Psi}^{\dagger} \boldsymbol{\Psi}_{\mathcal{Q}_{2}} \mathbf{h} .
\end{array}
$$

The application of Geršgorin's disc theorem [18] gives

$$
\begin{aligned}
& \theta_{\min , 1}\left\|\boldsymbol{\Psi}_{\mathcal{Q}_{1}} \mathbf{h}\right\|_{2}^{2} \leq\left\|\mathbf{A} \boldsymbol{\Psi}^{\dagger} \boldsymbol{\Psi}_{\mathcal{Q}_{1}} \mathbf{h}\right\|_{2}^{2} \leq \theta_{\max , 1}\left\|\boldsymbol{\Psi}_{\mathcal{Q}_{1}} \mathbf{h}\right\|_{2}^{2} \\
& \theta_{\min , 2}\left\|\boldsymbol{\Psi}_{\mathcal{Q}_{2}} \mathbf{h}\right\|_{2}^{2} \leq\left\|\mathbf{A} \boldsymbol{\Psi}^{\dagger} \boldsymbol{\Psi}_{\mathcal{Q}_{2}} \mathbf{h}\right\|_{2}^{2} \leq \theta_{\max , 2}\left\|\boldsymbol{\Psi}_{\mathcal{Q}_{2}} \mathbf{h}\right\|_{2}^{2}
\end{aligned}
$$

with $\theta_{\min , \ell} \triangleq \omega_{\min }^{2}\left(\mathbf{A}_{\ell} \boldsymbol{\Psi}_{\ell}^{\dagger}\right)-\mu_{\ell}\left(k_{\ell}-1\right)$ and $\theta_{\max , \ell} \triangleq$ $\omega_{\max }^{2}\left(\mathbf{A}_{\ell} \boldsymbol{\Psi}_{\ell}^{\dagger}\right)+\mu_{\ell}\left(k_{\ell}-1\right)$, for $\ell=1,2$.

In addition, the last term in (22) can be bounded as

$$
\begin{aligned}
& \left|\left(\mathbf{A} \boldsymbol{\Psi}^{\dagger} \boldsymbol{\Psi}_{\mathcal{Q}_{1}} \mathbf{h}\right)^{H} \mathbf{A} \boldsymbol{\Psi}^{\dagger} \boldsymbol{\Psi}_{\mathcal{Q}_{2}} \mathbf{h}\right| \\
& \quad \leq \sum_{i \in \mathcal{Q}_{1}, j \in \mathcal{Q}_{2}}\left|\left[\left(\mathbf{A} \boldsymbol{\Psi}^{\dagger}\right)^{H} \mathbf{A} \boldsymbol{\Psi}^{\dagger}\right]_{i, j}\left\|[\mathbf{\Psi} \mathbf{h}]_{i}\right\|[\mathbf{\Psi} \mathbf{h}]_{j}\right| \\
& \quad \leq \mu_{m}\left\|\boldsymbol{\Psi}_{\mathcal{Q}_{1}} \mathbf{h}\right\|_{1}\left\|\boldsymbol{\Psi}_{\mathcal{Q}_{2}} \mathbf{h}\right\|_{1} \\
& \quad \leq \mu_{m} \sqrt{k_{1} k_{2}}\left\|\boldsymbol{\Psi}_{\mathcal{Q}_{1}} \mathbf{h}\right\|_{2}\left\|\boldsymbol{\Psi}_{\mathcal{Q}_{2}} \mathbf{h}\right\|_{2} \\
& \quad \leq \frac{\mu_{m}}{2} \sqrt{k_{1} k_{2}}\left(\left\|\boldsymbol{\Psi}_{\mathcal{Q}_{1}} \mathbf{h}\right\|_{2}^{2}+\left\|\boldsymbol{\Psi}_{\mathcal{Q}_{2}} \mathbf{h}\right\|_{2}^{2}\right) \\
& \quad \leq \frac{\mu_{m}}{2} \sqrt{k_{1} k_{2}}\left\|\boldsymbol{\Psi}_{\mathcal{Q}} \mathbf{h}\right\|_{2}^{2},
\end{aligned}
$$


where (25a) follows from the definition of $\mu_{m}$, (25b) results from $\|\mathbf{u}\|_{1} \leq \sqrt{k}\|\mathbf{u}\|_{2}$, for $k$-sparse $\mathbf{u}$, and (25c) is a consequence of the arithmetic-mean geometric-mean inequality.

Combining (23), (24), and (25d) gives

$$
\theta_{\min }\left\|\boldsymbol{\Psi}_{\mathcal{Q}} \mathbf{h}\right\|_{2}^{2} \leq\left\|\mathbf{A} \boldsymbol{\Psi}^{\dagger} \Psi_{\mathcal{Q}} \mathbf{h}\right\|_{2}^{2} \leq \theta_{\max }\left\|\boldsymbol{\Psi}_{\mathcal{Q}} \mathbf{h}\right\|_{2}^{2},
$$

where $\theta_{\min } \triangleq \omega_{\min }^{2}-f\left(k_{1}, k_{2}\right), \theta_{\max } \triangleq \omega_{\max }^{2}+f\left(k_{1}, k_{2}\right), \omega_{\min } \triangleq$ $\omega_{\min }\left(\mathbf{A}_{1} \boldsymbol{\Psi}_{1}^{\dagger}, \mathbf{A}_{2} \boldsymbol{\Psi}_{2}^{\dagger}\right), \omega_{\max } \triangleq \omega_{\max }\left(\mathbf{A}_{1} \boldsymbol{\Psi}_{1}^{\dagger}, \mathbf{A}_{2} \boldsymbol{\Psi}_{2}^{\dagger}\right)$, and

$$
f\left(k_{1}, k_{2}\right) \triangleq \max \left\{\mu_{1}\left(k_{1}-1\right), \mu_{2}\left(k_{2}-1\right)\right\}+\mu_{m} \sqrt{k_{1} k_{2}} .
$$

Using the same steps as in (17a)-(17e), we get

$$
g\left(k_{1}, k_{2}\right)\left\|\boldsymbol{\Psi}_{\mathcal{Q}} \mathbf{h}\right\|_{2} \leq 2 \varepsilon \sqrt{\theta_{\max }}+2 \mu \sqrt{k}\left\|\boldsymbol{\Psi}_{\mathcal{S}^{c} \mathbf{X}}\right\|_{1},
$$

where $g\left(k_{1}, k_{2}\right) \triangleq \omega_{\min }^{2}-f\left(k_{1}, k_{2}\right)-\mu k$.

Next, we bound $g\left(k_{1}, k_{2}\right)$ from below by a function of $k=$ $k_{1}+k_{2}$. This can be done, e.g., by looking for the minimum [7]

$$
\hat{g}(k) \triangleq \min _{k_{1}: 0 \leq k_{1} \leq k} g\left(k_{1}, k-k_{1}\right)
$$

or equivalently

$$
\hat{g}(k) \triangleq \min _{k_{2}: 0 \leq k_{2} \leq k} g\left(k-k_{2}, k_{2}\right) .
$$

To find $\hat{g}(k)$ in (26) or in (27), we need to distinguish between two cases:

- Case 1: $\mu_{1}\left(k_{1}-1\right) \leq \mu_{2}\left(k_{2}-1\right)$

In this case, we get

$g\left(k-k_{2}, k_{2}\right)=\omega_{\min }^{2}-\mu_{2}\left(k_{2}-1\right)-\mu_{m} \sqrt{k_{2}\left(k-k_{2}\right)}-\mu k$.

A straightforward calculation reveals that the minimum of $g$ is achieved at

$$
k_{2}=\frac{k}{2}\left(1+\frac{\mu_{2}}{\sqrt{\mu_{2}^{2}+\mu_{m}^{2}}}\right)
$$

resulting in

$$
\hat{g}(k)=\omega_{\min }^{2}-\frac{1}{2}\left(\mu_{2}(k-2)+k \sqrt{\mu_{2}^{2}+\mu_{m}^{2}}\right)-\mu k .
$$

If $\hat{g}(k)>0$, then we have

$$
\left\|\mathbf{x}^{*}-\mathbf{x}\right\|_{2}=\|\mathbf{h}\|_{2} \leq C_{0} \varepsilon+C_{1}\left\|\Psi_{\mathcal{S}^{c}} \mathbf{x}\right\|_{1}
$$

where

$$
C_{0}=\frac{2 \sqrt{3}}{\sigma_{\min }(\boldsymbol{\Psi}) \hat{g}(k)}
$$

and

$$
C_{1}=\frac{1}{\sigma_{\min }(\boldsymbol{\Psi})}\left(\frac{2 \mu \sqrt{3 k}}{\hat{g}(k)}+\frac{1}{\sqrt{k}}\right) .
$$

Setting $\hat{g}(k)>0$ amounts to imposing

$$
k<\frac{2\left(1+\hat{\mu}_{2}\right)}{\hat{\mu}_{2}+2 \hat{\mu}_{\max }+\sqrt{\hat{\mu}_{2}^{2}+\hat{\mu}_{m}^{2}}},
$$

where we used Definitions 1 and 2 to get a threshold depending on the coherence parameters only.
- Case 2: $\mu_{2}\left(k_{2}-1\right) \leq \mu_{1}\left(k_{1}-1\right)$

Similarly to Case 1, we get

$$
\hat{g}(k)=\omega_{\min }^{2}-\frac{1}{2}\left(\mu_{1}(k-2)+k \sqrt{\mu_{1}^{2}+\mu_{m}^{2}}\right)-\mu k .
$$

If $\hat{g}(k)>0$, we must have

$$
k<\frac{2\left(1+\hat{\mu}_{1}\right)}{\hat{\mu}_{1}+2 \hat{\mu}_{\max }+\sqrt{\hat{\mu}_{1}^{2}+\hat{\mu}_{m}^{2}}} .
$$

Since $\hat{\mu}_{1} \leq \hat{\mu}_{2}$, by assumption, the inequality in (30) is tighter than the one in (29). We complete the proof by combining the thresholds in (19) and (29) to get (3).

\section{REFERENCES}

[1] R. Rubinstein, A. M. Bruckstein, and M. Elad, "Dictionaries for sparse representation modeling," Proc. IEEE, vol. 98, no. 6, pp. 1045-1057, Apr. 2010.

[2] O. Christensen, An Introduction to Frames and Riesz Bases, ser. Applied and Numerical Harmonic Analysis, J. J. Benedetto, Ed. Boston, MA, USA: Birkhäuser, 2002.

[3] M. Elad, Sparse and Redundant Representations - From Theory to Applications in Signal and Image Processing. New York, NY, USA: Springer, 2010.

[4] P. Milanfar and R. Rubinstein, "Analysis versus synthesis in signal priors," Inverse Problems, vol. 23, pp. 947-968, Jan. 2007.

[5] E. J. Candès, Y. C. Eldar, D. Needell, and P. Randall, "Compressed sensing with coherent and redundant dictionaries," Appl. Comput. Harmon. Anal., vol. 31, no. 1, pp. 59-73, Sep. 2011.

[6] E. J. Candès, J. Romberg, and T. Tao, "Stable signal recovery from incomplete and inaccurate measurements," Comm. Pure Appl. Math., vol. 59, no. 2 , pp. 1207-1223, Mar. 2005.

[7] C. Studer and R. Baraniuk, "Stable restoration and separation of approximately sparse signals," Appl. Comput. Harmon. Anal., submitted. [Online]. Available: http://arxiv.org/pdf/1107.0420v1.pdf

[8] S. G. Mallat, A Wavelet Tour of Signal Processing: The Sparse Way. Burlington, MA, USA: Academic Press, 2009.

[9] M. Elad, J.-L. Starck, P. Querre, and D. L. Donoho, "Simultaneous cartoon and texture image inpainting using morphological component analysis (MCA)," Appl. Comput. Harmon. Anal., vol. 19, no. 3, pp. 340-358, Jan. 2005.

[10] J. Fadili, J.-L. Starck, M. Elad, and D. L. Donoho, "MCALab: Reproducible research in signal and image decomposition and inpainting," Computing in Science \& Engineering, vol. 12, no. 1, pp. 44-63, Feb. 2010.

[11] J.-F. Cai, S. Osher, and Z. Shen, "Split Bregman methods and frame based image restoration," Multiscale Modeling \& Simulation, vol. 8, no. 2, pp. 337-369, Jan. 2010.

[12] C. Studer, P. Kuppinger, G. Pope, and H. Bölcskei, "Recovery of sparsely corrupted signals," IEEE Trans. Inf. Theory, vol. 58, no. 5, pp. 31153130, May 2012.

[13] G. Kutyniok, "Data separation by sparse representations," in Compressed Sensing: Theory and Applications, Y. C. Eldar and G. Kutyniok, Eds., New York, NY, USA: Cambridge University Press, 2012.

[14] Y. Liu, T. Mi, and S. Li, "Compressed sensing with general frames via optimal-dual-based $\ell_{1}$-analysis," IEEE Trans. Inf. Theory, submitted. [Online]. Available: http://arxiv.org/pdf/1111.4345.pdf

[15] S. Nam, M. Davies, M. Elad, and R. Gribonval, "The cosparse analysis model and algorithms," INRIA, Tech. Rep., Jun. 2011. [Online]. Available: http://arxiv.org/pdf/1106.4987v1.pdf

[16] L. Demanet and L. Ying, "Wave atoms and sparsity of oscillatory patterns," Appl. Comput. Harmon. Anal., vol. 23, no. 3, pp. 368-387, Jan. 2007.

[17] S. Becker, E. J. Candès, and M. Grant, "Templates for convex cone problems with applications to sparse signal recovery," in Mathematical Programming Computation, W. J. Cook, Ed., 2012, vol. 3, no. 3, pp. $165-218$.

[18] R. A. Horn and C. R. Johnson, Matrix Analysis. New York, NY, USA: Cambridge University Press, 1991.

[19] T. T. Cai, L. Wang, and G. Xu, "New bounds for restricted isometry constants," IEEE Trans. Inf. Theory, vol. 56, no. 9, pp. 1-7, Aug. 2010

[20] - ,Stable recovery of sparse signals and an oracle inequality," IEEE Trans. Inf. Theory, vol. 56, no. 7, pp. 3516-3522, Jul. 2010. 